\title{
Selective Sensing of Copper Ions by Mesoporous Porphyrinic Metal-Organic Frameworks Nanoovals
}

\author{
Zhiyuan Xu ${ }^{\S \sharp}$, QingYi Meng ${ }^{\S \sharp}$, Qiang Cao ${ }^{£}, \zeta$, Yushi Xiao ${ }^{£}, \zeta$, Huan Liu $^{£}$, Gang Han $^{£}$, Shuhua Wei ${ }^{\S}$, Jiang Yan ${ }^{\S}$,

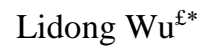 \\ ${ }^{£ \zeta}$ Key Laboratory of Control of Quality and Safety for Aquatic Products, Ministry of Agriculture, Chinese Academy \\ of Fishery Sciences, Beijing, 100141, China. \\ ${ }^{\S}$ School of Information science and technology, North China University of Technology, No. 5 Jinyuanzhuang \\ Street, Shijingshan District, Beijing 100144, China \\ $\zeta$ Shanghai Ocean University, Shanghai 201306, China
}

Table of Content

1. The photograph of the reaction product of PCN222. After reaction, the color change from green to purple.

2. (A) Nitrogen adsorption-desorption isotherm of PCN222. (B) The corresponding BarrettJoyner-Halenda (BJH) pore size distribution of PCN222.

3. (A) SEM image of PCN222, (B) Dynamic light scattering (DLS) analysis of PCN222 and (C) the UV/Vis spectroscopy of different sizes PCN222.

4. (A) CV of bare GC, Chitosan/GC, and Chitosan-PCN222/GC electrodes in $5 \mathrm{mmol} \mathrm{\textrm {L } ^ { - 1 }}$ $\mathrm{K}_{3} \mathrm{Fe}(\mathrm{CN})_{6} / \mathrm{K}_{4} \mathrm{Fe}(\mathrm{CN})_{6}$, (B) Nyquist plots of bare GC, Chitosan/GC, and Chitosan-PCN222/GC electrodes in $5 \mathrm{mmol} \mathrm{L}^{-1} \mathrm{~K}_{3} \mathrm{Fe}(\mathrm{CN})_{6} / \mathrm{K}_{4} \mathrm{Fe}(\mathrm{CN})_{6}$, (C) $\mathrm{CV}$ of the Chitosan-PCN222/GC electrode with $1 \mu \mathrm{mol} \mathrm{L}-1$ of $\mathrm{Cu}^{2+}$, and (D) SEM image of Chitosan-PCN222/GC electrode.

5. Amperometric response of PCN222 based sensor without (the black pillar) and with (the red pillar) $1 \mu \mathrm{mol} \mathrm{L}-1 \mathrm{Cu}^{2+}$ in the presence of interfered metal ions.

6. Amperometric response of Chitosan-PCN222(50 $\mu \mathrm{g}) / \mathrm{GC}$ with successive addition of $1 \mu \mathrm{mol}$ $\mathrm{L}^{-1} \mathrm{Cu}^{2+}$. (B) The typical amperometric current response curves of the Chitosan-PCN222(5 $\mu \mathrm{g}) / \mathrm{GC}$, the Chitosan-PCN222(500 ng)/GC and the Chitosan/GC electrodes (Blank) with successive addition of $10 \mu \mathrm{L} \mathrm{Cu}^{2+}$ solution into $10 \mathrm{~mL}$ PBS solution. Applied potential: $-0.1 \mathrm{~V}$ versus $\mathrm{Ag} / \mathrm{AgCl}$. (C) The corresponding calibration curves of steady-state currents versus concentrations of $\mathrm{Cu}^{2+}$ : the Chitosan-PCN222(5 $\left.\mu \mathrm{g}\right) / \mathrm{GC}$, the Chitosan-PCN222(500 ng)/GC and the Chitosan/GC electrodes (Blank).

\footnotetext{
${ }^{\#}$ Equal contribution to this work. ${ }^{*}$ Corresponding author.Tel/Fax: 86-10-68690712; E-mail: lidongwu@mit.edu
} 
7. (A) X-ray photoelectron spectroscopy of PCN222 and PCN222-Cuㄹ ${ }^{2+}$, and (B) TEM image of PCN222 binding of $\mathrm{Cu}^{2+}$.

8. After electrochemical measurements, the Chitosan-PCN222/GC sensor incubates in $50 \mu \mathrm{mol} \mathrm{L}$ ${ }^{1}$ EDTA for 30 minutes, and then detect $1 \mu \mathrm{mol} \mathrm{L}^{-1}$ of $\mathrm{Cu}^{2+}$ for stability tests.

9. After incubation of the PCN222 in $\mathrm{H}_{2} \mathrm{O}$, methanol, ethanol, acetone and acetonitrile for 30 minutes, the response signal of the Chitosan-PCN222/GC sensor with $1 \mu \mathrm{mol} \mathrm{L}{ }^{-1}$ of $\mathrm{Cu}^{2+}$.

10. Previously reported various sensors for $\mathrm{Cu}^{2+}$.
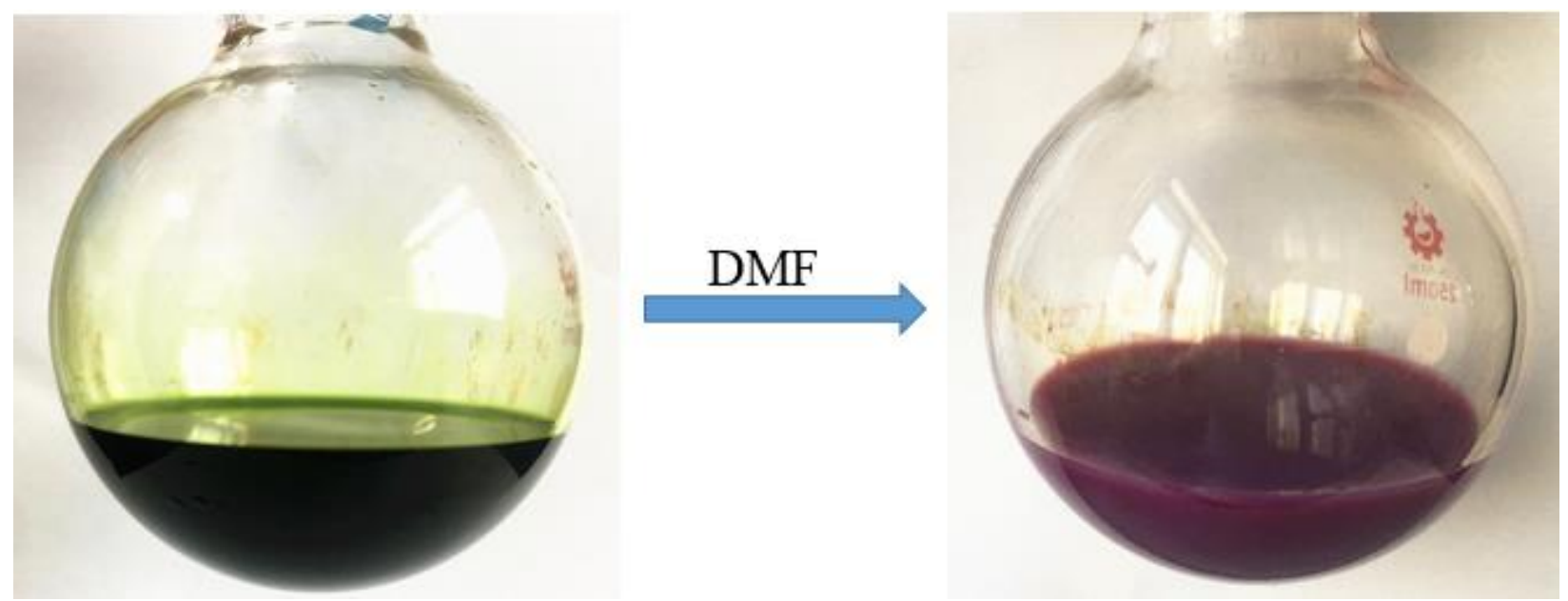

Figure S1. The photograph of the reaction product of PCN222. After reaction, the color change from green to purple. 

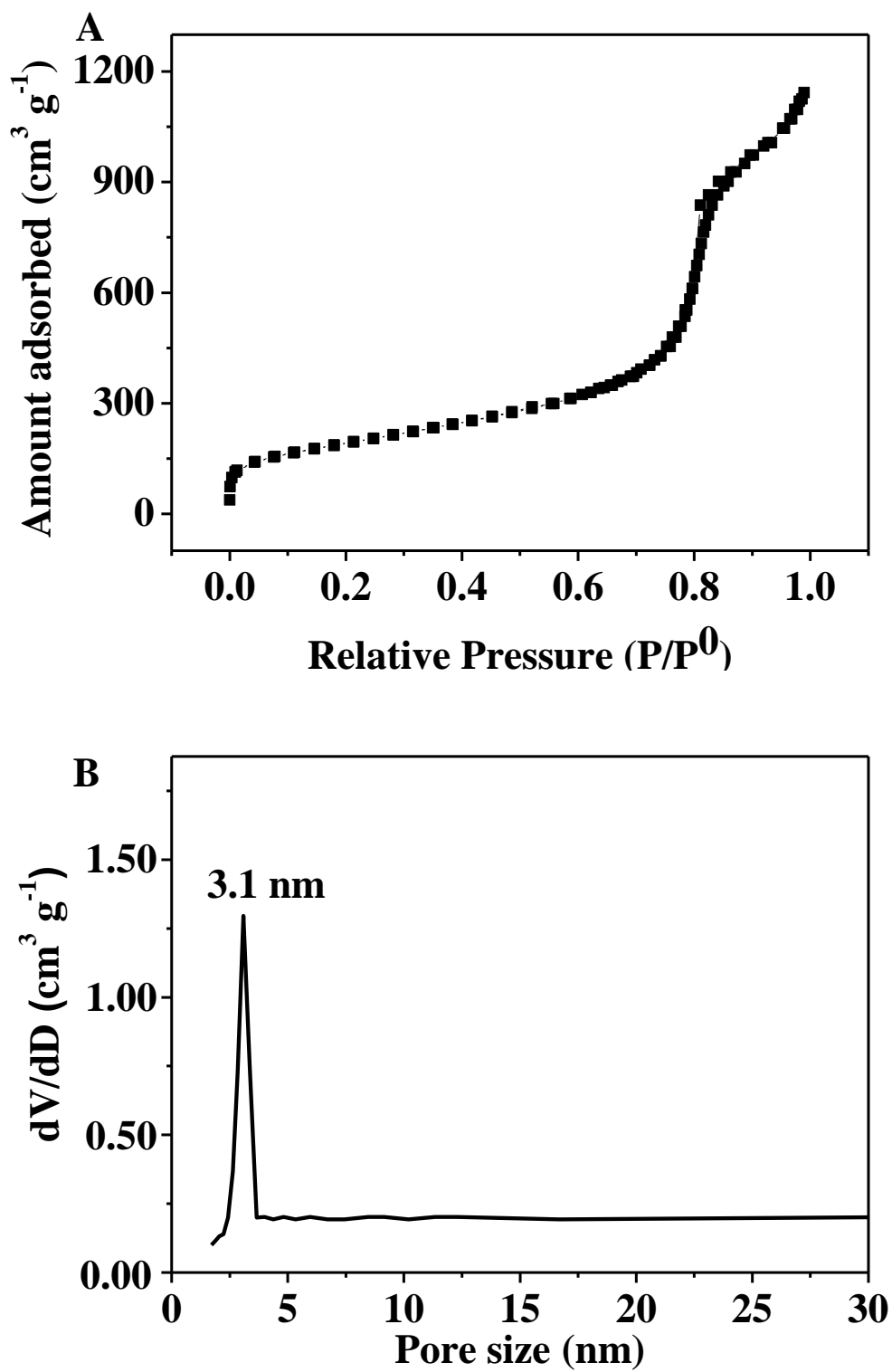

Figure S2. (A) Nitrogen adsorption-desorption isotherm of PCN222. (B) The corresponding Barrett-Joyner-Halenda (BJH) pore size distribution of PCN222. 

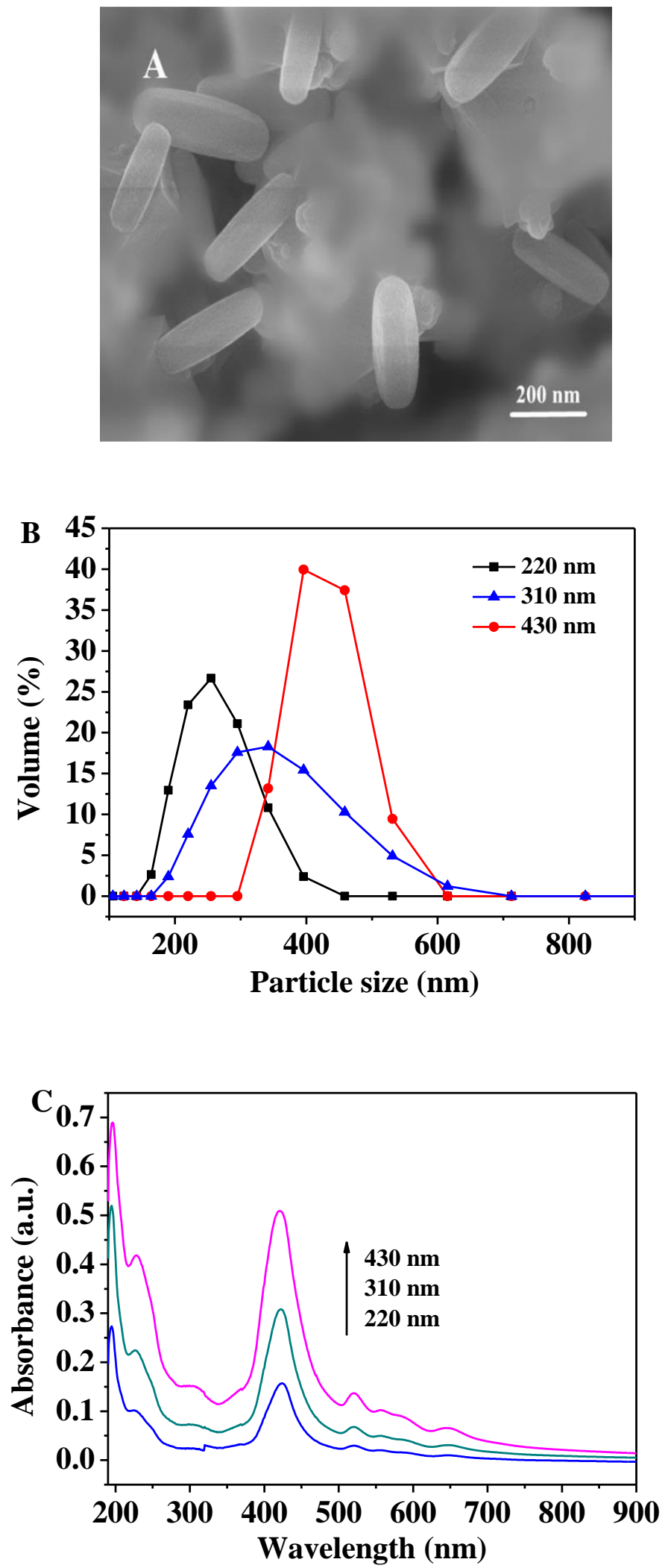

Figure S3. (A) SEM image of PCN222, (B) Dynamic light scattering (DLS) analysis of PCN222 and (C) the UV/Vis spectroscopy of different sizes PCN222. 

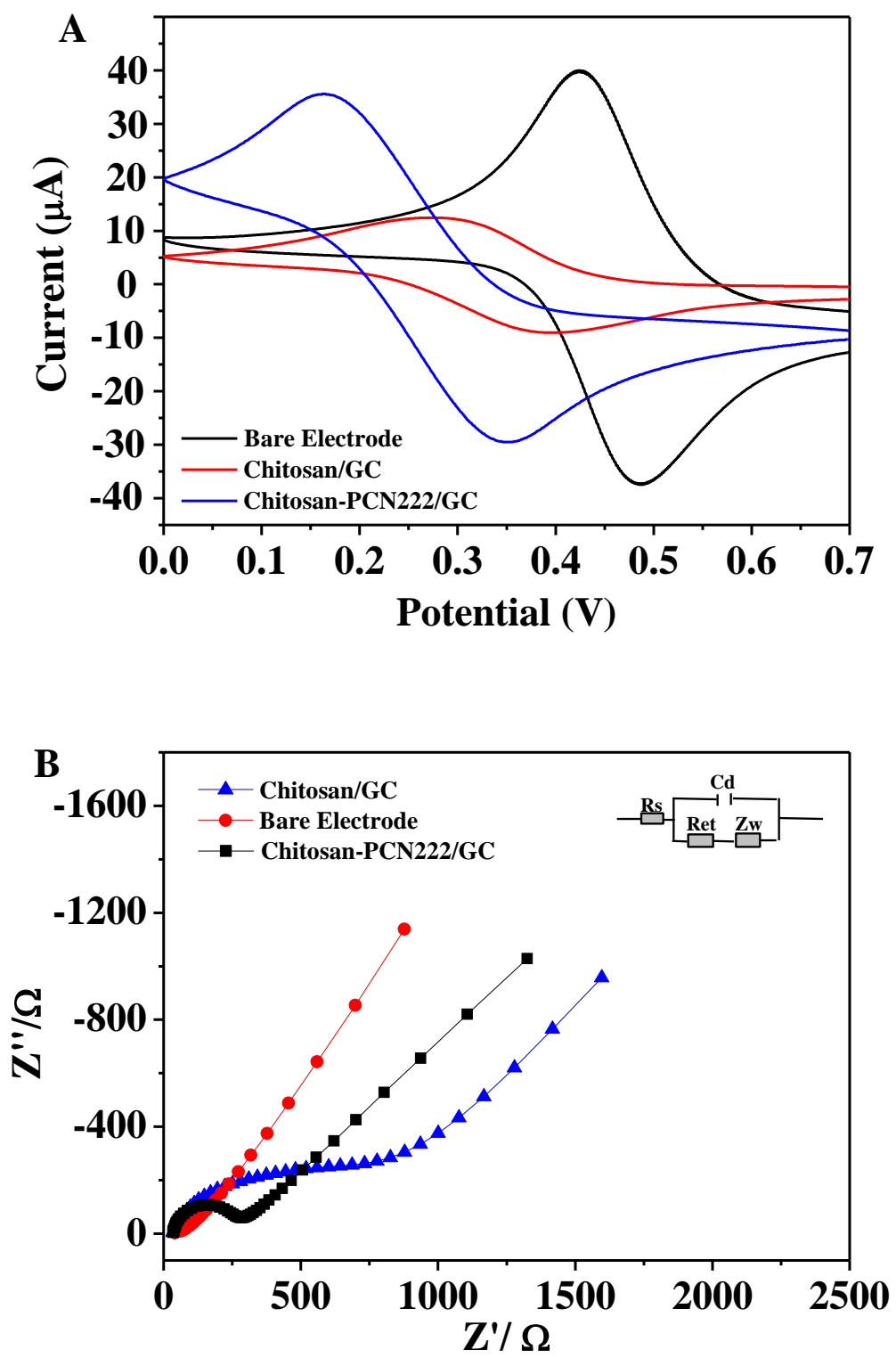

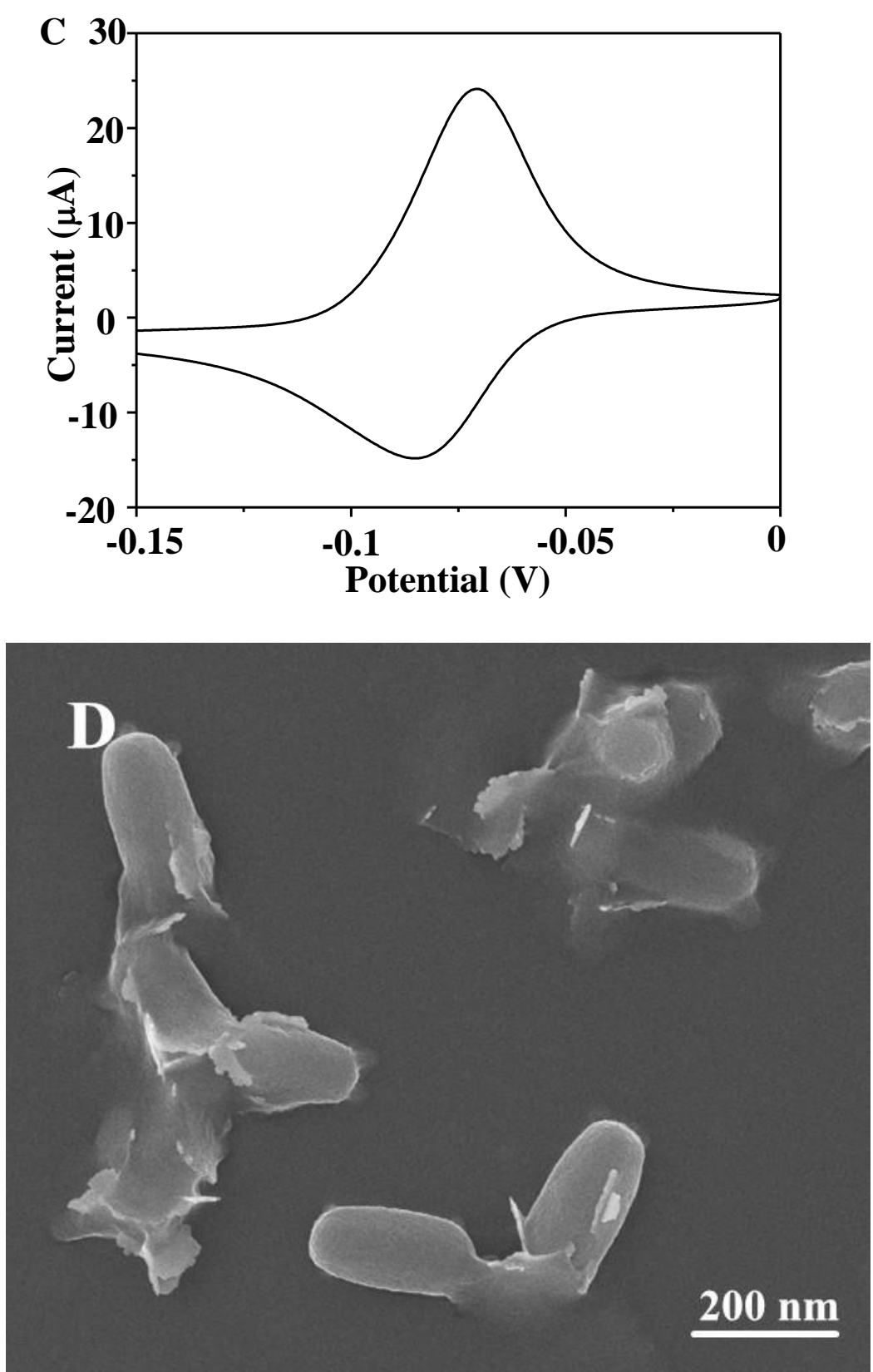

Figure S4. (A) CV of bare GC, Chitosan/GC, and Chitosan-PCN222/GC electrodes in 5 mmol L${ }^{1} \mathrm{~K}_{3} \mathrm{Fe}(\mathrm{CN})_{6} / \mathrm{K}_{4} \mathrm{Fe}(\mathrm{CN})_{6}$, (B) Nyquist plots of bare GC, Chitosan/GC, and Chitosan-PCN222/GC electrodes in $5 \mathrm{mmol} \mathrm{L}^{-1} \mathrm{~K}_{3} \mathrm{Fe}(\mathrm{CN})_{6} / \mathrm{K}_{4} \mathrm{Fe}(\mathrm{CN})_{6}$, (C) $\mathrm{CV}$ of the Chitosan-PCN222/GC electrode with $1 \mu \mathrm{mol} \mathrm{L}^{-1}$ of $\mathrm{Cu}^{2+}$, and (D) SEM image of Chitosan-PCN222/GC electrode. 


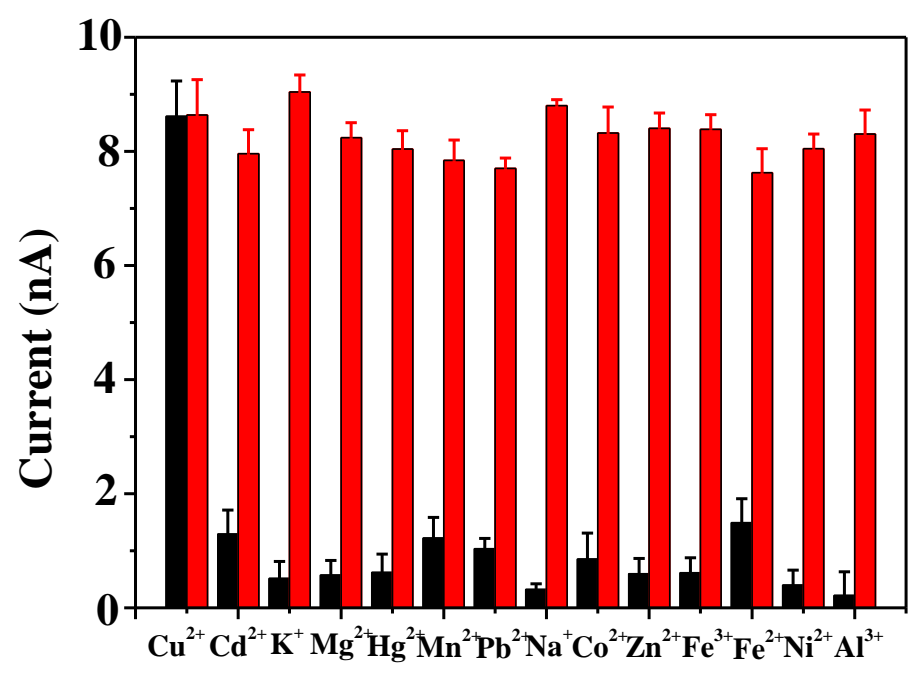

Figure S5. Amperometric response of PCN222 based sensor without (the black pillar) and with (the red pillar) $1 \mu \mathrm{mol} \mathrm{L}{ }^{-1} \mathrm{Cu}^{2+}$ in the presence of interfered metal ions.

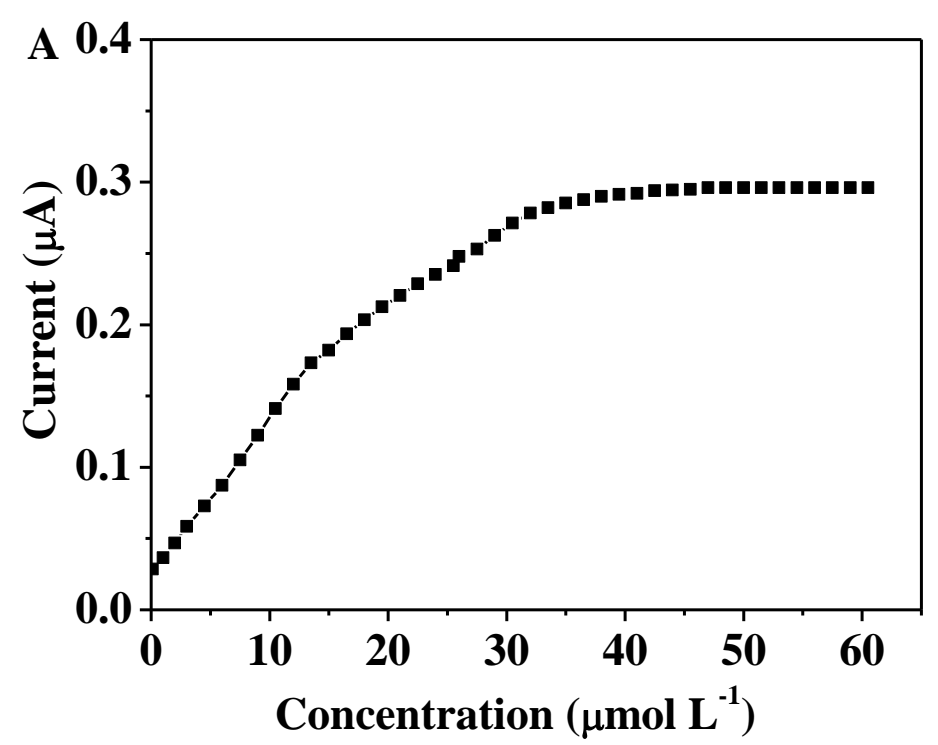



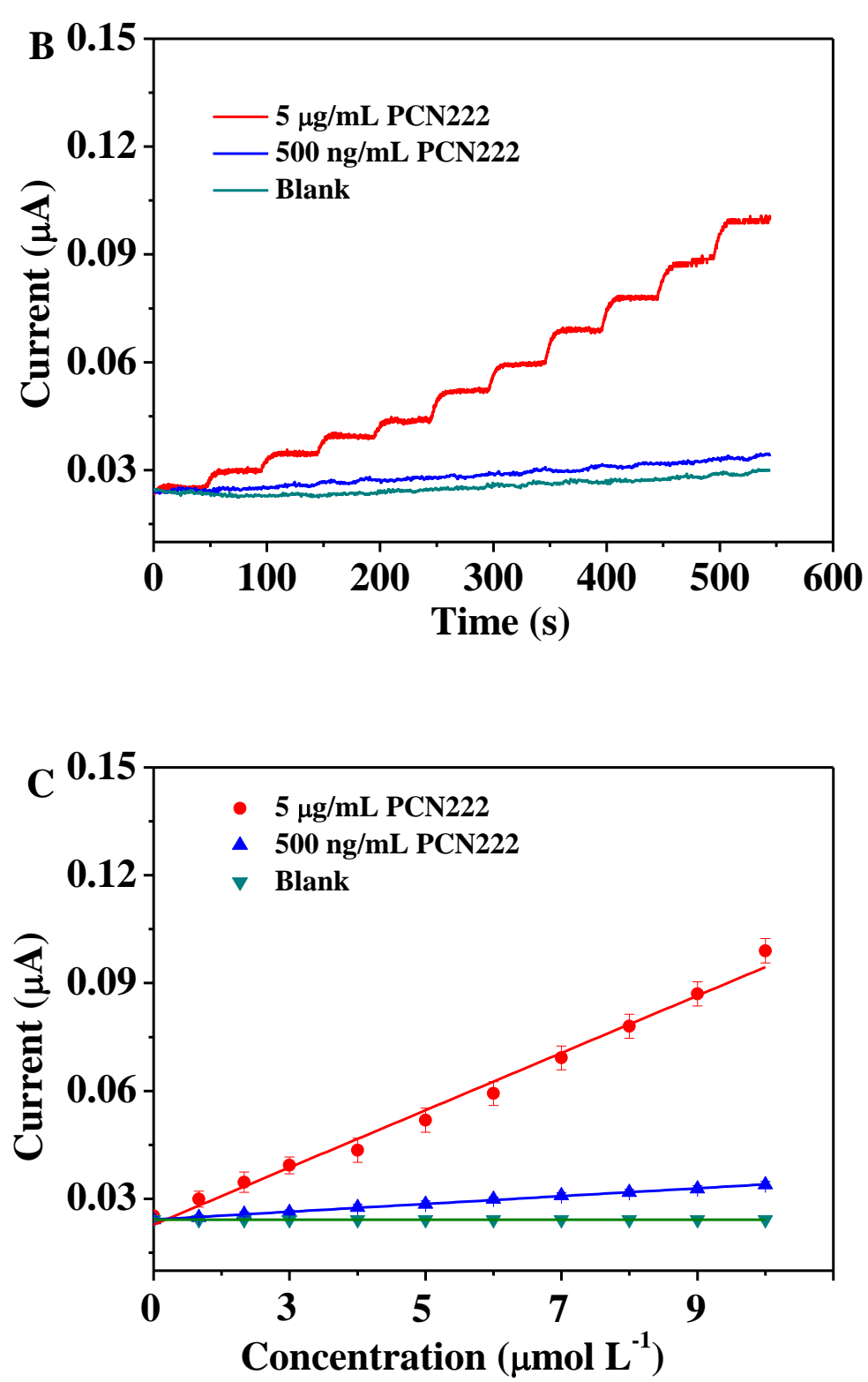

Figure S6. (A) Amperometric response of Chitosan-PCN222(50 $\mu \mathrm{g}) / \mathrm{GC}$ with successive addition of $1 \mu \mathrm{mol} \mathrm{L}{ }^{-1} \mathrm{Cu}^{2+}$. (B) The typical amperometric current response curves of the ChitosanPCN222(5 $\mu \mathrm{g}) / \mathrm{GC}$, the Chitosan-PCN222(500 ng)/GC and the Chitosan/GC electrodes (Blank) with successive addition of $10 \mu \mathrm{L} \mathrm{Cu}{ }^{2+}$ solution into $10 \mathrm{~mL}$ PBS solution. Applied potential: -0.1 $\mathrm{V}$ versus $\mathrm{Ag} / \mathrm{AgCl}$. (C) The corresponding calibration curves of steady-state currents versus concentrations of $\mathrm{Cu}^{2+}:$ the Chitosan-PCN222(5 $\left.\mu \mathrm{g}\right) / \mathrm{GC}$, the Chitosan-PCN222(500 ng)/GC and the Chitosan/GC electrodes (Blank). 

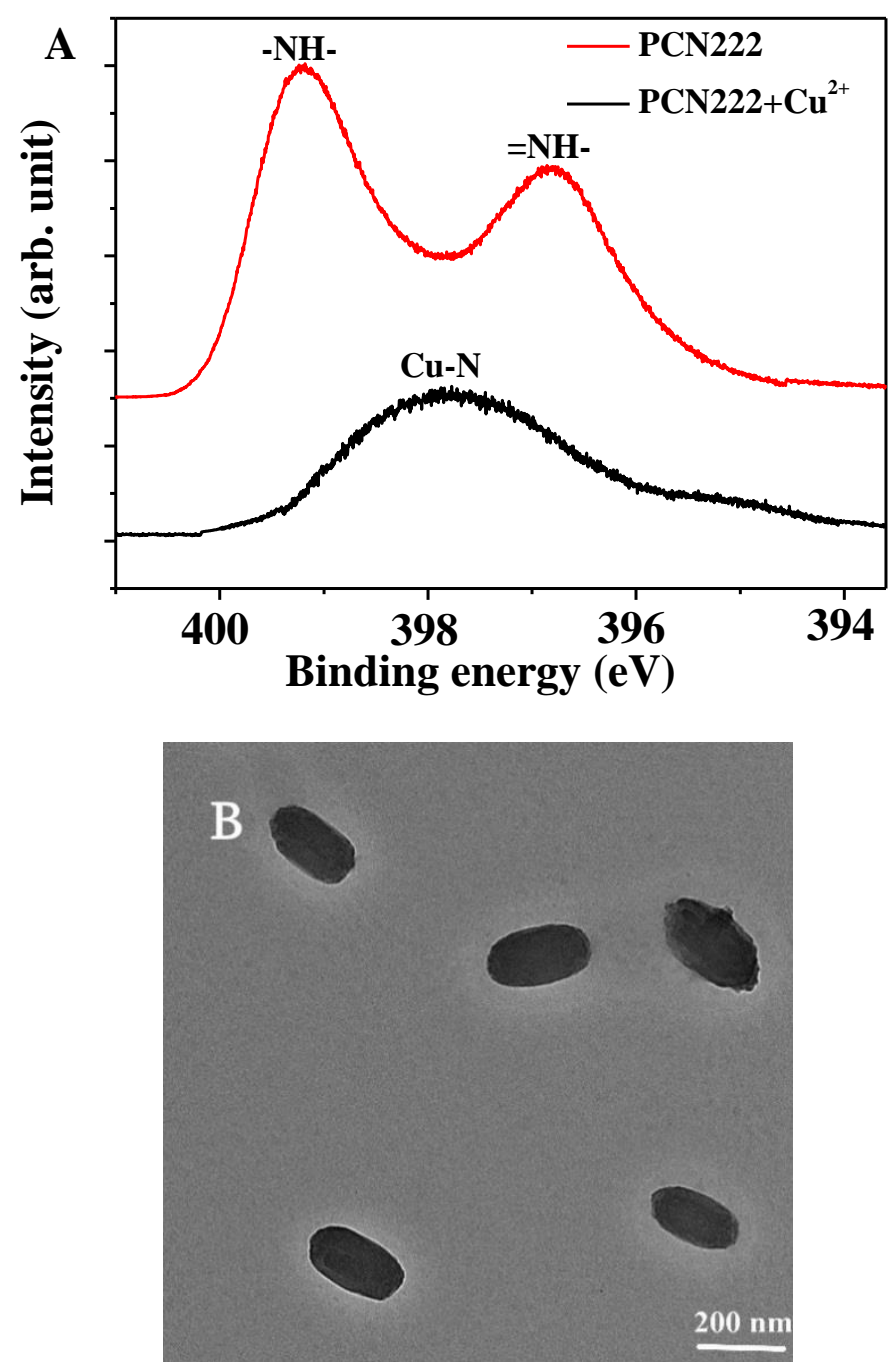

Figure S7. (A) X-ray photoelectron spectroscopy of PCN222 and PCN222-Cu ${ }^{2+}$, and (B) TEM image of PCN222 binding of $\mathrm{Cu}^{2+}$. 


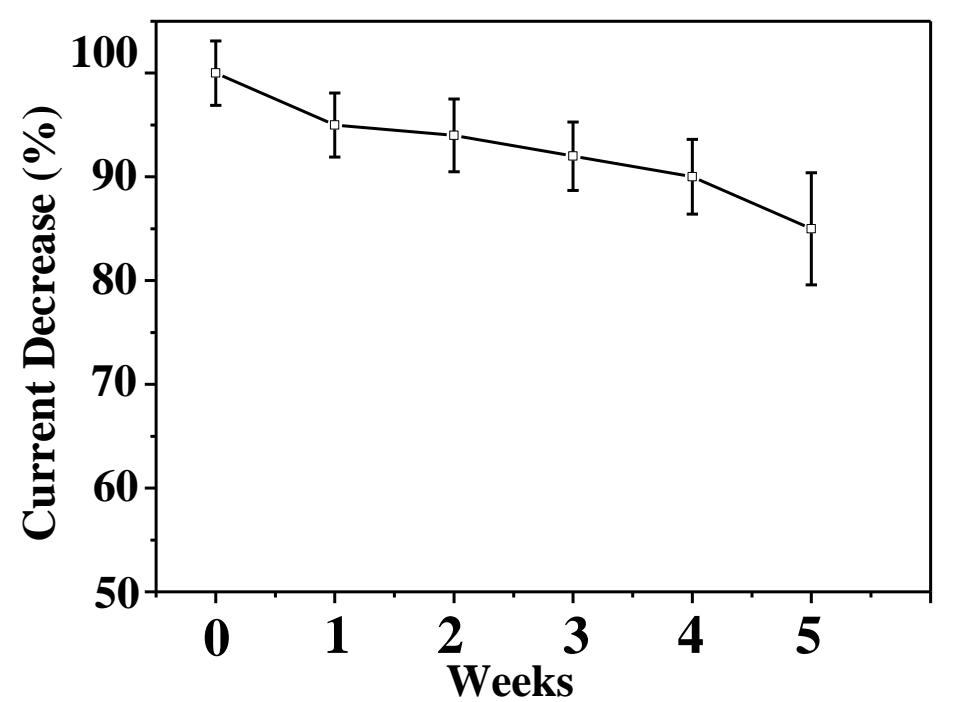

Figure S8. After electrochemical measurements, the Chitosan-PCN222/GC sensor incubates in 50 $\mu$ mol L ${ }^{-1}$ EDTA for 30 minutes, and then detect $1 \mu \mathrm{mol} \mathrm{L}^{-1} \mathrm{of} \mathrm{Cu}^{2+}$ for stability tests.

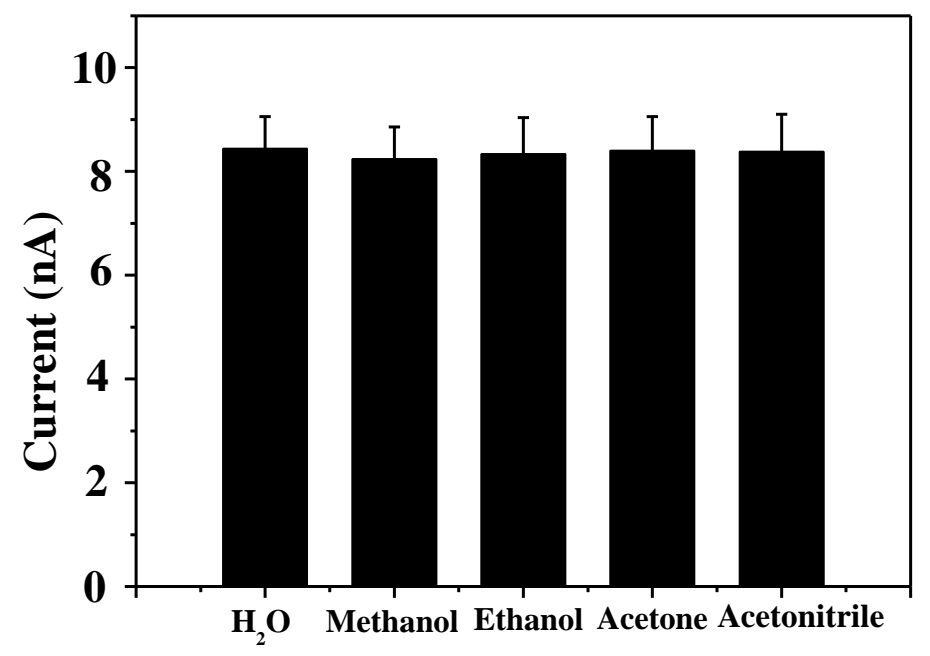

Figure S9. After incubation of the $\mathrm{PCN} 222$ in $\mathrm{H}_{2} \mathrm{O}$, methanol, ethanol, acetone and acetonitrile for 30 minutes, the response signal of the Chitosan-PCN222/GC sensor with $1 \mu \mathrm{mol} \mathrm{L}^{-1}$ of $\mathrm{Cu}^{2+}$. 
Table S1. Previously reported various sensors for $\mathrm{Cu}^{2+}$

\begin{tabular}{ccc}
\hline Sensors & LOD & Reference \\
\hline PCN222 & $50 \mathrm{nM}$ & This work \\
MOF-525 & $67 \mathrm{nM}$ & 1 \\
$\mathrm{Fe}_{3} \mathrm{O}_{4}$-MWCNT & $5.3 \mu \mathrm{M}$ & 2 \\
Hydrazine & $50 \mathrm{nM}$ & 3 \\
\hline
\end{tabular}

1. Song, Z.; Fan, G. C.; Li, Z. M.; Gao, F. X.; Luo, X. L. Anal Chem 2018, 90, 10681-10687.

2. Wang, T. Y.; Viennois, E.; Merlin, D.; Wang, G. L. Anal Chem 2015, 87, 8173-8180.

3. Xianyu, Y. L.; Zhu, K.; Chen, W. W.; Wang, X. F.; Zhao, H. M.; Sun, J. S.; Wang, Z.; Jiang, X. Y. Anal Chem 2013, 85, 7029-7032. 